\title{
Optimization of Complex Geometry Using Tenth Order Partial Differential Equation
}

\author{
Syed Khawar Nadeem Kirmani* and Raja Noshad Jamil \\ School of Science, Department of Mathematics, \\ University of Management and Technology, Lahore, Pakistan \\ *khawar.kirmani@umt.edu.pk
}

\begin{abstract}
This paper presents an efficient and intuitive technique of shape parameterization for design optimization using a partial differential equation (PDE) of order ten. It shows how the choice of two introduced parameters can enable one to parameterize complex geometries. With the use of PDE based formulation, it is shown in this paper how the shape can be defined and manipulated on the basis of parameterization and the boundary value approaches by which complex shapes can be created. Further the boundary conditions which are used in it are a boundary and an intermediate curves for defining the shape. This technique allows complex shapes to be parameterized intuitively using a very small set of design parameters. Hence, Practical design optimization of problems becomes more achievable by applying PDE based approach of shape parameterization by incorporating standard numerical optimization techniques [1,2].
\end{abstract}

Keywords: PDE surfaces, smoothness, continuity, ten boundary curves

\section{Introduction}

Parameterizing the shape of objects is one of the essential factors in practical design optimization. Embellishing a general explanation for objects, in which values of design parameters determine their shape is rudimentary in parameterization $[3,4]$. By designating a particular value for a specific item from this general explanation, one can create a new design each time with its application congruent to its own properties.

From previous literature about shape optimization, the vital factor emerging is the selection of design variables and their shape parameterization [5, 6]. Selection of multiple variables at a time can cause chaotic implications including mismanagement of computational time, whereas choosing few variables may produce limited results [7, 8]. Hence, using shapes with few parameters was considered more practical method in optimization, where the parameters must be able to intuitively define the predictable effect on the surface of shape.

Previous researches showcased nodal coordinate approach as a primitive method of shape optimization, where node coordinates of the discrete finite- 
element model calibrated as design variables [1]. This approach proved ineffective for a large number of design variables optimization. Mesh parameterization approach [9], the use of solid modeling [10] and the natural design variable method [11] were developed to overcome the drawbacks of this basic method. However, these methods proved to be costly and often produced restricted optimization results. Spline approach particularly Bezier and B-Splines, became the more advanced method for optimization where a series of polynomial functions defined any shape. This approach suggested that a surface should be represented as a mesh of rectangular curvilinear regions where a set of control points would define the shape [12]. It depicted that a large number of shape parameters and complex geometry was a setback for this approach.

The aim of this paper is to optimize a parametric shape using minimum shape constraints based on $10^{\text {th }}$ order partial differential equations (PDE) with 10 boundary conditions [7]. This technique follows a boundary-value approach where an object's shape can consists of surface patches bounded by character curves. Additionally, a small number of shape parameters proficiently define the surface's shape which facilitates the process of optimization both locally and globally.

The outline of the paper depicts the following,

- The study illustrates how a parametric surface can be created by PDE method.

- A methodology underpinning proficient parameterization of PDE surfaces is also catered in this paper.

- Various examples of design optimization is also a focus of this paper.

\section{PDE Surfaces}

PDE method for blend generation was first introduced by Bloor and Wilson [13,1] in computer aided geometric design (CAGD).

Assume a parametric function $\varphi(s, t)$ giving the surface in $E^{3}$ where $s$ and $t$

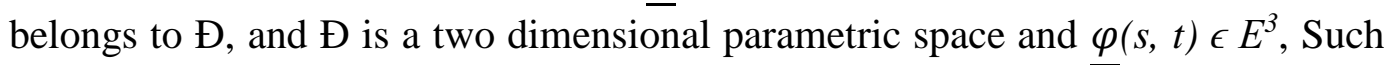
that $\mathfrak{R}^{2}(\mathrm{Ð}) \rightarrow \mathrm{E}^{3}$. The parametric form of the function is: $\underline{\varphi}(s, t)=(x(s, t), y(s, t)$, $z(s, t))$ The PDE equation used for this work is:

$$
\left(\frac{\partial^{2}}{\partial s^{2}}+\lambda^{2} \frac{\partial^{2}}{\partial t^{2}}\right)^{5} \underline{\varphi}(s, t)=0
$$

where $\lambda$ is called a smoothing parameter. Various solutions can be derived for equation (1). Bloor and Wilson (1996) outlined the most accepted method where solution of the above equation for desired optimum PDE surface can be formulated 
by boundary conditions. Additionally, the desired geometric shape is acquired by considering the vital shape parameters $[7,13,14]$.

The choice of parametric domain and boundary conditions pivot the shape of surface for acquiring solution of equation (1). Here $Đ$ is usually considered to be a rectangle such that, $Ð$ : $s_{0} \leq s \leq s_{1}, t_{0} \leq t \leq t_{1}$ [3,9]. For optimizing the final shape of a model, parameter $\lambda$ also plays a vital role.

Usually, the calculation of $x, y$ and $z$ coordinates with a parameter $\lambda$ which is normally constant but for this paper, we can optimize it according to the desired shape and can mold the model as well. Since, the PDE in equation (1) is of tenth order; hence, ten boundary conditions are requisite for the solution. Here, ten positional curves are considered as ten boundary conditions. Let $Đ$ be a finite domain defined as $Đ: 0 \leq s \leq 1,0 \leq t \leq 2 \pi$ such that:

$$
\begin{aligned}
& \underline{\varphi}(0, t)=\underline{f_{0}}(t), \\
& \underline{\varphi}\left(k_{1}, t\right)=\underline{f}_{k_{1}}(t), \\
& \underline{\varphi}\left(k_{2}, t\right)=\underline{f}_{k_{2}}(t), \\
& \underline{\varphi}\left(k_{3}, t\right)=\underline{f}_{k_{3}}(t), \\
& \underline{\varphi}\left(k_{4}, t\right)=\underline{f}_{k_{4}}(t), \\
& \underline{\varphi}\left(k_{5}, t\right)=\underline{f}_{k_{5}}(t), \\
& \underline{\varphi}\left(k_{6}, t\right)=\underline{f}_{k_{6}}(t), \\
& \underline{\varphi}\left(k_{7}, t\right)=\underline{f}_{k_{7}}(t), \\
& \underline{\varphi}\left(k_{8}, t\right)=\underline{f}_{k_{8}}(t), \\
& \underline{\varphi}(1, t)=\underline{f}_{1}(t) .
\end{aligned}
$$

Where $\underline{f}_{0}(t), \underline{f}_{k_{1}}(t), \underline{f}_{k_{2}}(t), \underline{f}_{k_{3}}(t), \underline{f}_{k_{4}}(t), \underline{f}_{k_{5}}(t), \underline{f}_{k_{6}}(t), \underline{f}_{k_{7}}(t), \underline{f}_{k_{8}}$ $(t)$, and $f_{1}(t)$ are given ten conditions. The unknowns $k_{1}, k_{2}, k_{3}, k_{4}, k_{5}, k_{6}, k_{7}$ and $k_{8}$ in above group of equations belong to $(0,1)$, such that:

$$
0<k_{1}<k_{2}<k_{3}<k_{4}<k_{5}<k_{6}<k_{7}<k_{8}<1
$$

These unknown also reflect the parameters of optimization and may also adjudicate the relative position of intermediate curves. By slightly altering these parameters, one can optimize the geometric shape as well. Shape can be locally controlled by the boundary curves. Therefore, they also locally optimize the final shape. 
Solution of equation (1) is based upon method of separation of variables. $\varphi(s, t)=$

$$
\underline{\alpha}_{0}(s)+\sum_{n=1}^{N}\left[\underline{\beta}_{n}(s) \cos (n t)+\underline{\gamma}_{n}(s) \sin (n t)\right],
$$

where

$$
\begin{aligned}
& \underline{\alpha}_{0}=\underline{\alpha}_{00}+\underline{\alpha}_{01} s+\underline{\alpha}_{02} s^{2}+\underline{\alpha}_{03} s^{3}+\underline{\alpha}_{04} s^{4}+\underline{\alpha}_{05} s^{5}+\underline{\alpha}_{06} s^{6}+\underline{\alpha}_{07} s^{7}+ \\
& \underline{\alpha}_{08} s^{8}+\underline{\alpha}_{09} s^{9} \\
& \underline{\beta}_{n}=\underline{\beta}_{n 1} e^{a n s}+\underline{\beta}_{n 2} e^{-a n s}+\underline{\beta}_{n 3} s e^{a n s}+\underline{\beta}_{n 4} s e^{-a n s}+\underline{\beta}_{n 5} s^{2} e^{a n s}+ \\
& \underline{\beta}_{n 6} s^{2} e^{-a n s}+\underline{\beta}_{n 7} s^{3} e^{a n s}+c \underline{\beta}_{n 8} s^{3} e^{-a n s}+\underline{\beta}_{n 9} s^{4} e^{a n s}+\underline{\beta}_{n 10} s^{4} e^{-a n s} \\
& \underline{\gamma}_{n}=\underline{\gamma}_{n 1} e^{a n s}+\underline{\gamma}_{n 2} e^{-a n s}+\underline{\gamma}_{n 3} s e^{a n s}+\underline{\gamma}_{n 4} s e^{-a n s}+\underline{\gamma}_{n 5} s^{2} e^{a n s}+ \\
& \underline{\gamma}_{n 6} s^{2} e^{-a n s}+\underline{\gamma}_{n 7} s^{3} e^{a n s}+\underline{\gamma}_{n 8} s^{3} e^{-a n s}+\underline{\gamma}_{n 9} s^{4} e^{a n s}+\underline{\gamma}_{n 10} s^{4} e^{-a n s}
\end{aligned}
$$

and where all the vector valued constants $\alpha_{i}$ 's, $\beta_{i}$ 's and $\gamma_{i}$ 's can be determined by the given boundary conditions. The final shape of the geometry can be manipulated by these boundary conditions as shown in figure 1 .

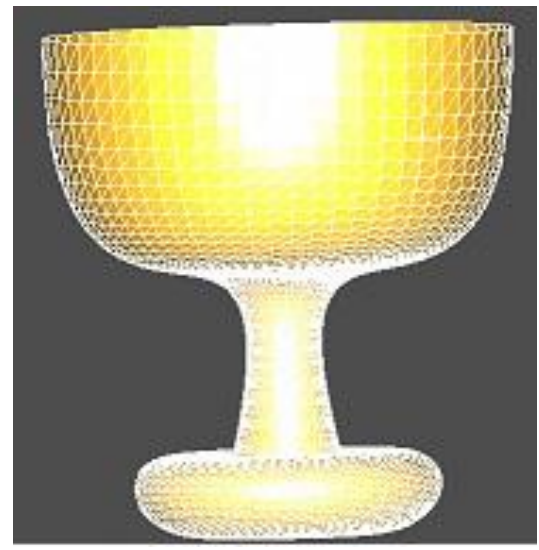

Figure 1. Optimum PDE surface

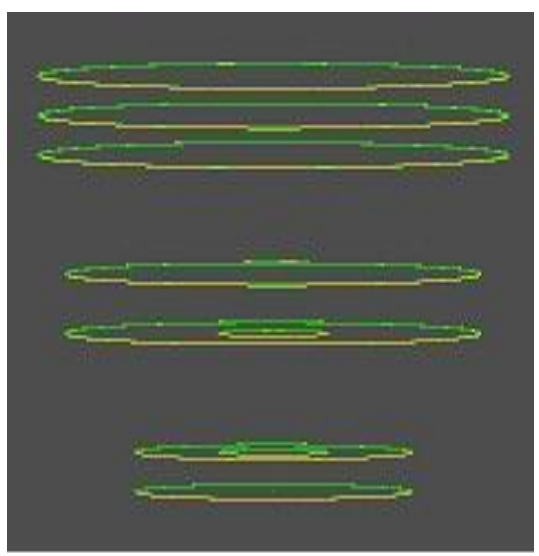

Figure 2. Ten positional curves

Figure 2 shows the ten positional curves as boundary conditions. Figure 1 is the end product by using tenth order PDE. The parameters $k_{1}, k_{2}, k_{3}, k_{4}, k_{5}, k_{6}$, $k_{7}$ and $k_{8}$ are the shape parameters together with " $a$ " as shown in equation (1) which depicts how these parameters are affect the final shape and also illustrate the manipulation of shape by readjustment of their position. Since these parameters have a range of 0 to 1 , therefore, all parameters can have values between 0 and 1 . For a particular choice of $k$ 's let's assume the following values:

Table 1. Assigned values of shape parameters

\begin{tabular}{|l|l|l|l|l|l|l|l|}
\hline$k_{1}$ & $k_{2}$ & $k_{3}$ & $k_{4}$ & $k_{5}$ & $k_{6}$ & $k_{7}$ & $k_{8}$ \\
\hline
\end{tabular}




\begin{tabular}{|l|l|l|l|l|l|l|l|}
\hline 0.1 & 0.2 & 0.3 & 0.4 & 0.6 & 0.7 & 0.8 & 0.9 \\
\hline
\end{tabular}

Figure 1 is the outcome of the values shown in table 1. For instance, the shape in figure 2 will change if $k_{4}$ and $k_{5}$ are increased to 0.45 and 0.65 respectively.

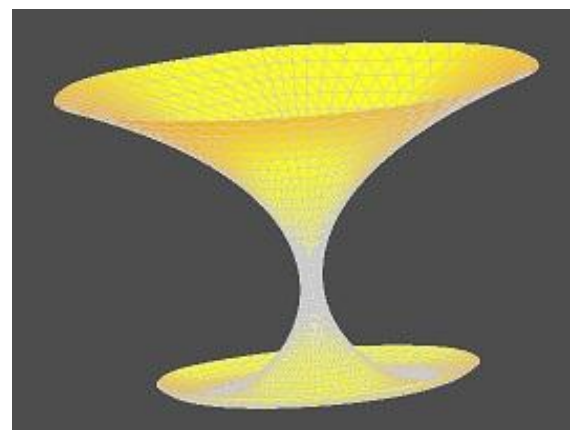

Figure 3. Shape parameter $k_{4}=4.5$

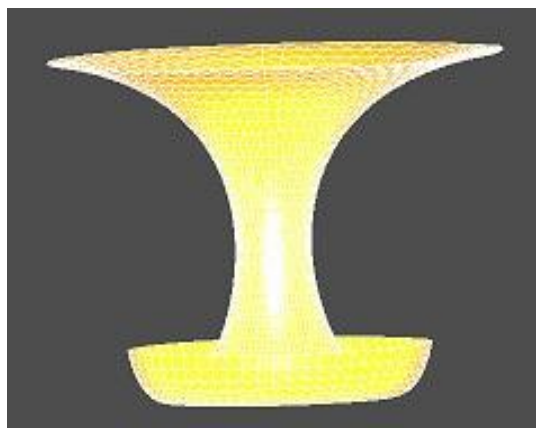

Figure 4. Shape parameter $k_{5}=6.5$

The final shape will take the form as shown in Figure 5.

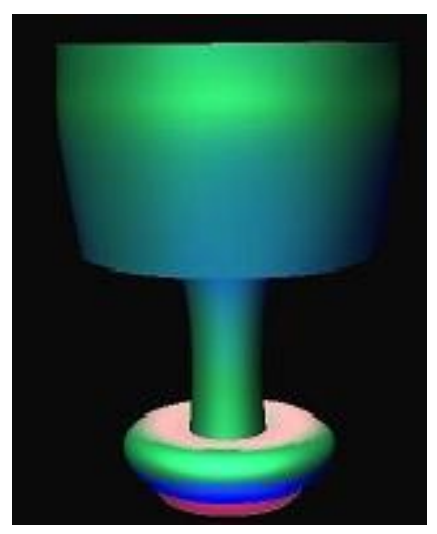

Figure 5. Shape with changed $k_{4}$ and $k_{5}$

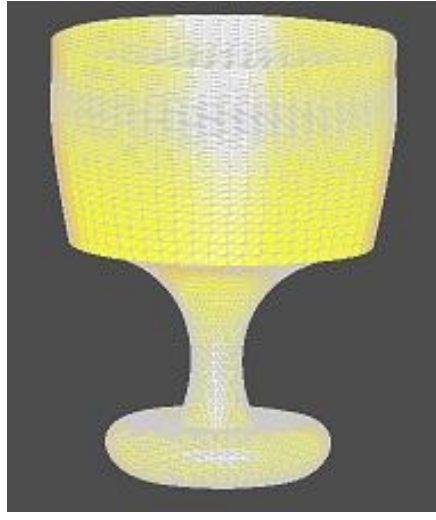

Figure 6. $k_{7}$ is chnaged from 0.8 to 0.85

In Figure $6, k_{7}$ is slightly changed from 0.8 to 0.85 . Hence, one can manipulate the given geometry according to desired outcome.

Another benefit of involving more curves is that we can localy control the shape of geometry. Following is a simple demonstration of the generated surface using PDE of tenth order. 

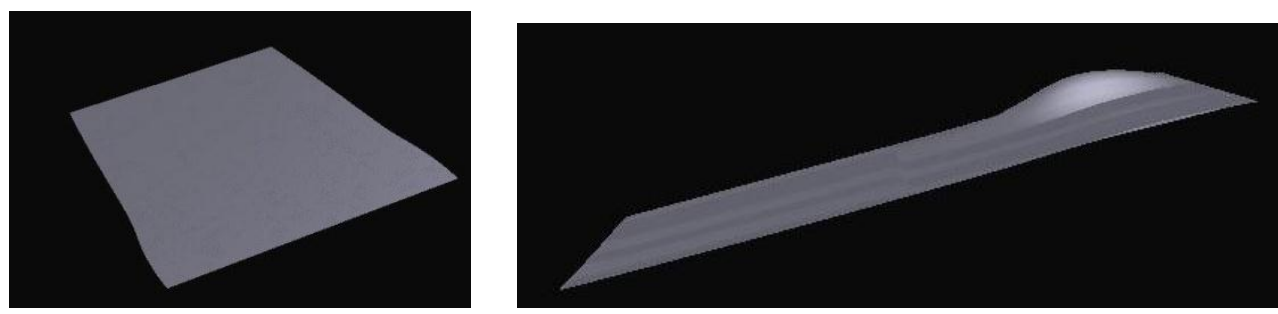

Figure 7. PDE plane surface from $\mathrm{z}=0$ to $\mathrm{z}=1$

Figure 8. Ninth boundry curve, z-coordinate of a point

Figure 7 represents a plane surface generated by eqution (1) along with conditions (2) to (11). In Figure 8, only one point is changed from $\mathrm{z}=0$ to $\mathrm{z}=1$ of curve number nine. The result shows that we can locally change the shape without altering the whole geometry.

In real world, an insect bite may affects a particular part of the human skin which may cause that bitten part of skin to swell locally without tearing the whole skin; a shape can be optimized in the similar fashion.

\section{Error Analysis}

It is essential that for a certain set of boundary conditions, various Fourier coefficients must be analyzed .The approximate solution can be as follows;

$$
\underline{\Psi}(s, t)=\underline{\alpha}_{0}(s)+\sum_{n=1}^{N}\left[\underline{\beta}_{n}(s) \cos (n t)+\underline{\gamma}_{n}(s) \sin (n t)\right]+\underline{E R R O R}(s, t)
$$

where $\mathrm{N}$ reflects the number of Fourier modes. As $\mathrm{N}$ is a finite value, so an error term is rudimentary which is called remainder $\underline{E R R O R}(s, t)$. This can be created in the following way, where coefficients $\beta(s)$ and $\gamma(s)$ are determined by amplitudes of the nth mode in boundary conditions.

The term $\operatorname{ERROR}(s, t)$ also denotes utilizing high frequency modes to the surface. Due to the finite value of N, this term effects the entire shape of the surface. The remainder term is defined as:

$$
\begin{gathered}
\underline{\operatorname{ERROR}}(s, t)=\underline{\rho}_{1}(t) e^{w s}+\underline{\rho}_{2}(t) e^{-w s}+\underline{\rho}_{3}(t) s e^{w s}+\underline{\rho}_{4}(t) s e^{-w s}+ \\
\underline{\rho}_{5}(t) s^{2} e^{w s}+\underline{\rho}_{6}(t) s^{2} e^{-w s}+\underline{\rho}_{7}(t) s^{3} e^{w s}+\underline{\rho}_{8}(t) s^{3} e^{-w s}+\underline{\rho}_{9}(t) s^{4} e^{w s}+ \\
\underline{\rho}_{10}(t) s^{4} e^{-w s}
\end{gathered}
$$

where $\underline{\varrho}_{1}, \varrho_{2}, \varrho_{3}, \varrho_{4}, \varrho_{5}, \varrho_{6}, \varrho_{7}, \varrho_{8}, \varrho_{9}, \varrho_{10}$ and $w$ are acquired by regarding a disparity between original boundary conditions and the boundary conditions fulfilled by function, where the original boundary conditions are the chosen curves which satisfy equation (1):

$$
\underline{x}(s, t)=\underline{\alpha}_{0}(s)+\sum_{n=1}^{N}\left[\underline{\beta}_{n}(s) \cos (n t)+\underline{\gamma}_{n}(s) \sin (n t)\right]
$$


where, $\beta(s)$ and $\gamma(s)$ are achieved from the aforementioned Fourier analysis of the boundary conditions. The ten conditions $\underline{\xi_{0}}, \xi_{k_{1}}, \xi_{k_{2}}, \xi_{k_{3}}, \xi_{k_{4}}, \xi_{k_{5}}, \xi_{k_{6}}, \xi_{k_{7}}$, $\xi_{k_{8}}$ and $\xi_{1}$ are the difference between the original boundary conditions and the boundary conditions fulfilled by $\underline{X}(s, t)$, with

$$
\begin{aligned}
& \underline{\xi_{0}}=\underline{f_{t}}-\underline{x}(0, t) \\
& \underline{\xi_{k_{1}}}=\underline{f_{t}}-\underline{x}\left(k_{1}, t\right) \\
& \underline{\xi_{k_{2}}}=\underline{f_{t}}-\underline{x}\left(k_{2}, t\right) \\
& \underline{\xi_{k_{3}}}=\underline{f_{t}}-\underline{x}\left(k_{3}, t\right) \\
& \underline{\xi_{k_{4}}}=\underline{f_{t}}-\underline{x}\left(k_{4}, t\right) \\
& \underline{\xi_{k_{5}}}=\underline{f_{t}}-\underline{x}\left(k_{5}, t\right) \\
& \underline{\xi_{k_{6}}}=\underline{f_{t}}-\underline{x}\left(k_{6}, t\right) \\
& \underline{\xi_{k_{7}}}=\underline{f_{t}}-\underline{x}\left(k_{7}, t\right) \\
& \underline{\xi_{k_{8}}}=\underline{f_{t}}-\underline{x}\left(k_{8}, t\right) \\
& \underline{\xi_{1}}=\underline{f_{t}}-\underline{x}(1, t)
\end{aligned}
$$

With these conditions $\underline{\operatorname{ERROR}}(\mathrm{s}, \mathrm{t})$ can be obtained.

The constant $\mathrm{w}$ in equation (15) is selected from the plausible proximity of approximate surface to desired surface. For this, we select $\mathrm{N}$ as an equal to $\lambda(\mathrm{N}+$ 1) which is adjacent to the actual decay rate of difference $[\underline{\Psi}(s, t)-\underline{x}(s, t)][7]$. The choice of $\mathrm{N}$ plays a vital role in error terms that is, an increase in the value of $\mathrm{N}$ causes a decrease in the error $\underline{E R R O R}(s, t)$. For a good approximation $N \geq 6$ is a superlative choice which minimize the error $\underline{\operatorname{ERROR}}(s, t)$.

\section{Conclusion}

The determination, manipulation or optimization of a geometric surface requires certain boundary conditions. The manipulation of these conditions particularly the eight parameters $k_{1}, k_{2}, k_{3}, k_{4}, k_{5}, k_{6}, k_{7}$ and $k_{8}$ along with ' $a$ ' will aid in the development of 3D geometry. The current paper explains the generation of PDE surfaces via ten curves as boundary conditions. The paper also discusses how an optimum surface can be achieved by the flexibility of the method, even when a surface is either close or an open surface. The study also exemplifies how a surface can be manipulated by the readjustment of the boundary conditions, where eight parameters called as shape parameters have been introduced to discuss how effectively they can change the final shape of a model. 


\section{References}

[1] Warren J, Weimer H. Subdivision methods for geometric design: A constructive approach. Burlington: Morgan Kaufmann publisher, 1995.

[2] Zhang JJ, You LH. Surface representation using second, fourth and mixed order partial differential equations. International Conference on Shape Modeling and Applications, Genova, Italy, May 7-11, 2001.

[3] Zhang JJ, You L. PDE based surface representation vase design. Comput Graphics. 2002;26: 89-98.

[4] Faux ID, Pratt MJ. Computational geometry for design and manufacture. New York: Halsted Press; 1979.

[5] Yamashina H, Fukushima K, Saijo A. CAD for free form surfaces. Camput Integr Manuf Syst. 1996;9(1): 9-18.

[6] Davis P. Interpolation and Approximation. New York: Dover Publications; 1975.

[7] Piegl L. On NURBS: A Survey. IEEE Comput Graphics Appl. 1991;11(1): 5571.

[8] Bloor MIG, Wilson MJ. Generating blend surfaces using partial differential equation. Comput Aided Des. 1989;21(3); 165-171.

[9] Ugail H, Bloor MIG, Wilson MJ. Techniques for interactive design using the PDE method. ACM Trans Graphics. 1999;18: 195-212.

[10] Piegl L. Recursive algorithms for the representation of parametric curves and surfaces. Comput Aided Design. 1985;17(5); 225-229.

[11] Schumaker LL. Spline functions: Basic theory. New York: John Wiley and Sons; 1981.

[12] Bloor MIG, Wilson MJ. Using partial differential equations to generate free form surfaces. Comput Aided Des. 1990;22(4): 202-212.

[13] Du H, Qin H. Direct manipulation and interactive sculpting of PDE surfaces: Proceeding of Eurographics 2000, Interlaken, Switzerland. Comput Graphics Forum. 2000;19: 261-270.

[14] Ugail H, Bloor MIG, Wilson, MJ. Manipulations of PDE surfaces using an interactively defined parameterisation. Comput Graphics. 1999;24(3): 525-534. 